

\section{MÉLANGES de l'Université Saint-Joseph}

Volume LXV - 2013-2014

Résumés/Abstracts

ÉTHIQUE, POLITIQUE ET RHÉTORIQUE DANS LES TRAITÉS ET LEURS COMMENTAIRES, DE L'ANTIQUité À LA RENAISSANCE. ORIENT ET OCCIDENT

Actes du séminaire tenu en 2012, UPR 76 (CNRS, Paris)

Présentation

Frédérique WOERTHER

Du neuf sur l'Alexandros chrétien?

Pierre CHIRON

Ethical and Political Commentary on Aristotle's Rhetoric

During the Renaissance

Lawrence D. GREEN

Moral Virtue in Late Antique Platonism.

Some Elements of a Background to Ethics in Early Arabic Philosophy Dominic O'Meara

Rhetoric Across Cultures: The Case of Themistius

John W. WATT

Alfarabi (870-950): Reason, Revelation, and Politics

Charles E. ButTerworth

The Influence of the Nicomachean Ethics on Medieval Jewish Thought Steven HARVEY 
Le commentaire sur l'Éthique à Nicomaque dans l'Occident latin.

Un genre, une pratique ( $\mathrm{XIII}^{\mathrm{e}}-\mathrm{XV}^{\mathrm{e}}$ siècle)

Bénédicte SÈrE

Scientia moralis and prudentia.

The Medieval Roots of a Contemporary Problem

Alexander FIDORA

*

Al-Fārābī's Introduction to the Five Rational Arts with Reference to

The Five Aphorisms (al-Fușūl al-Khamsa) and Ibn Bājja's Comments (Ta 'ālīq)

Terence J. KLEVEN

Un fragment retrouvé (sur la composition des éléments) du troisième Livre perdu du Grand commentaire au De Caelo par Averroès

Teymour Morel et Maroun AouAD

Culture vs. Civilization

Ibn Khaldūn's Muqaddima and the Literal Meaning of 'Umrān

Waseem EL-RAYES

Inventaire des marques lapidaires médiévales du Liban

Patricia ANTAKI-MAsson

OBJETS ET DOCUMENTS DIVERS PROVENANT DES FOUILLES DE JEAN MÉcÉRIAN DANS L'ANTIOCHÈnE (1932-1939)

Avant-propos

Lévon Nordiguian

Présentation

Lévon Nordiguian et Tomasz WALISZEWSKI

Le père Jean Mécérian (1888-1965)

Bibliographie sélective du père Jean Mécérian (1888-1965) 
Le Sanctuaire de Saint-Syméon-Stylite-le-Jeune au Mont Admirable à la lumière de la documentation photographique du père Jean Mécérian Mariusz GwIAZDA

Dix siècles d'une collection levantine.

Les sculptures de la collection du père Jean Mécérian

Dagmara WielgosZ-RonDOLINO

Lampes à huile de la collection du père Jean Mécérian

Tomasz WALISZEWSKI

Objets métalliques de la collection du père Jean Mécérian

Agnieszka SzULC-KaJAK

An Overview of the Medieval Pottery From the Antiochene Region

Excavated by Jean Mécérian

Tasha Vorderstrasse

Les miscellanea de la collection du père Jean Mécérian

Mariusz GwIAZDA

Nous remercions vivement Bassam Kahwagi, Roupène Basmadjian, Harouth Bezdjian, Christia Sayegh et Karam Hoyek pour avoir gracieusement mis à notre disposition leur temps et leur talent pour les retouches, le calibrage, le détourage et le scannage des illustrations reproduites dans ce volume. (E.G.) 


\title{
Le Sanctuaire de Saint-Syméon-Stylite-le-Jeune au Mont Admirable à la lumière de la documentation photographique du père Jean Mécérian
}

\author{
Mariusz GwIAZDA
}

L'article retrace les étapes de l'évolution et de la transformation de l'architecture du sanctuaire et du monastère au Mont Admirable, situé entre Antioche sur l'Oronte et Séleucie de Piérie. Son histoire est liée à la personne de Syméon Stylite le Jeune qui vécut au $\mathrm{VI}^{\mathrm{e}}$ siècle de notre ère et qui, sur le site en question, fonda un monastère avec ses disciples. À la différence de nombreux monastères et sanctuaires de la période protobyzantine, les informations sur ce site, proviennent, hormis les ruines, des récits des vies de Syméon et de sa mère sainte Marthe, ainsi que du grand nombre de textes historiques ${ }^{1}$, contenant des renseignements importants sur les communautés monastiques qui habitèrent les lieux. Ces sources nous livrent également des informations de grande valeur sur l'architecture du sanctuaire, et permettent ainsi de mieux comprendre les changements survenus.

Les premières fouilles archéologiques sur le site du sanctuaire furent menées sous la direction du père Jean Mécérian dans les années 1932-1938. Le déroulement et les résultats de ses travaux furent présentés dans quelques brefs rapports de fouilles et complétés par la publication d'un petit nombre d'objets sélectionnés². Malgré le

\footnotetext{
VAN DEN VEN P. (1962-1970), La vie ancienne de S. Syméon Stylite le Jeune (521-592), 2 vol., vol. I: Introduction et texte grec - vol. II: Vie grecque de sainte Marthe mère de S. Syméon, (Subsidia Hagiographica, 32) Société des Bollandistes, Bruxelles.

2 Millet G. (1933), «La mission archéologique du P. Mécérian dans l'Antiochène », Comptes rendus des Séances de l'Académie des Inscriptions et Belles-Lettres 77/3, p. 343-348; id. (1935), «Un rapport du R.P. Mécérian sur les fouilles au monastère de Saint-Syméon-le Jeune au Mont Admirable (Syrie) », Comptes rendus des Séances de l'Académie des Inscriptions et Belles-Lettres 79/2, p. 195-197; id. (1936), «Rapport du P. Mécérian sur les fouilles au monastère de Saint-Syméon le Jeune au Mont Admirable (Syrie) », Comptes rendus des Séances de l'Académie des Inscriptions et Belles-Lettres 80/3, p. 205-206; MÉcÉRIAN J. (1934), « Une mission archéologique dans l'Antiochène. Rapport sur la deuxième campagne de fouilles 1933 », Comptes rendus des Séances de l'Académie des Inscriptions et Belles-Lettres 78/2, p. 144-149; id. (1948), "Monastère de Saint-Siméon-Stylite-le-Jeune, exposé des fouilles », Comptes rendus des Séances de l'Académie des Inscriptions et Belles-Lettres 92/3, p. 323-328; id. (1951), «Le monastère de Saint Syméon le Stylite du Mont Admirable ", in Actes du VI Congrès international des études byzantines, Paris 27 juillet - 2 août 1948, 2 vol. en 1, École des Hautes Études, Paris, vol. II,
} 
nombre d'années écoulées depuis la fin du travail de terrain, les résultats définitifs des recherches du R.P. Mécérian n'ont pu être élaborés avant sa mort en 1965. En 19631965, les fouilles sur le site furent reprises par Wachtang Djobadze. Ses travaux se limitèrent, dans une grande mesure, au nettoyage et à la documentation des parties du sanctuaire déjà explorées par son prédécesseur. Peu après les fouilles de Wachtang Djobadze, des recherches sur le site nettoyé furent poursuivies par Jacqueline Lafontaine-Dosogne (1965). C'est à ces deux chercheurs que nous devons les plus importantes publications, comprenant de vastes descriptions de l'architecture du sanctuaire, établies après confrontation avec les sources écrites ${ }^{3}$.

Cette réflexion sur la transformation de l'architecture du sanctuaire de SaintSyméon-le-Stylite prend pour point de départ la documentation photographique établie par le père Jean Mécérian lors des fouilles qu'il entreprit au cours des années trente du $\mathrm{XX}^{\mathrm{e}}$ siècle. Ce fonds se trouve actuellement à la Bibliothèque Orientale de l'Université Saint-Joseph de Beyrouth. Il est composé de 4000 négatifs et clichés ${ }^{4}$. Pour les besoins de notre analyse, nous avons essentiellement choisi des photos représentant des constructions qui furent déplacées, lors des fouilles, afin de mettre au jour les toutes premières étapes de la construction des édifices, et qui ne figurent

p. 299-302; id. (1962), « Les inscriptions du Mont Admirable », Mélanges de l'Université Saint Joseph 38, p. 297-330.; id. (1964), «Expédition archéologique dans l'Antiochène occidentale », MUSJ 40, p. 46-48.

3 Djobadze W. (1964a), « Report on Archaeological Activities in the Vicinity of Antakya », Türk Arkeoloji Dergisi 13/1, p. 53-55; id. (1964b), «Second Preliminary Report on Excavations in the Vicinity of Antioch-on-the-Orontes », Türk Arkeoloji Dergisi 13/2, p. 32-35; id. (1965), « Vorläufiger Bericht über Grabungen und Untersuchungen in der Gegend von Antiochia am Orontes », Istanbuler Mitteilungen 15, p. 218-242; id. (1976), Materials for the Study of Georgian Monasteries in the Western Environs of Antioch on the Orontes, (Corpus Scriptorium Christianorum Orientalium. Subsidia 48) Secrétariat du Corpus SCO, Louvain; id. (1986), Archaeological Investigations in the Region West of Antioch onthe-Orontes, (Forschungen zur Kunstgeschichte und Christlichen Archäologie, 13) Franz Steiner Verlag Wiesbaden, Stuttgart; LAFontaine-Dosogne J. (1967), Itinéraires archéologiques dans la région d'Antioche. Recherches sur le monastère et sur l'iconographie de S. Syméon Stylite le Jeune, (Bibliothèque de Byzantion, 4) Édition de Byzantion, Bruxelles. Cf. Peeters P. (1928), «L'église Géorgienne du Clibanion au Mont Admirable», Analecta Bollandiana 46, p. 241-286; vAn DEN Ven P. (1961), «Le martyrium en triconque dans la vie de Sainte Marthe », Byzantion 31, p. 249-255; id., La vie ancienne de S. Syméon Stylite le Jeune (521-592), vol. I: Introduction et texte grec, p. 191-221; Verzone P. (1974), «Il santuario di S. Simeone il Giovane sul Monte Delle Meraviglie », Corsi di cultura sull 'arte Ravennate e Bizantina, p. 273-286; CALlot O. (1989), « À propos de quelques colonnes de stylites syriens », in Etienne R., Le Dinahet M.-T. et Yon M. (éds.), Architecture et poésie dans le monde grec: Hommage à Georges Roux, (Collection de la Maison de l'Orient Méditerranéen, Sér. Arch., 11) Maison de 1'Orient Méditerranéen, Paris, p. 107-122; Morray D.W. (1994), « The Defences of the Monastery of St Simeon the Younger on Samandağ », Orientalia Christiana Periodica 60/2, p. 619-623; REY-CoQUAIs J.-P. (1995), «Inscriptions du Mont Admirable », MUSJ 52, p. 199-226.

4 Je tiens à remercier M. Lévon Nordiguian, conservateur de la photothèque de la Bibliothèque Orientale à l'Université Saint-Joseph de Beyrouth, de m'avoir facilité l'accès aux archives et de m'avoir autorisé à les utiliser pour les besoins du présent article. Mes remerciements vont également à Joanna Pazio, Sławek Polaczek, Marcin Romaniuk et Krzysztof Stasiak, étudiants de 1'Université de Varsovie chargés des scans de la documentation photographique. 





ni sur les plans du site, ni dans les rapports préliminaires de fouilles. Cette documentation permettra donc de réviser et de compléter l'état actuel des connaissances sur le sujet.

Avant de passer à la présentation des sources photographiques, nous ferons le point sur la représentation actuelle de l'évolution du sanctuaire de Saint-Syméonle-Stylite, basée sur les résultats de fouilles antérieures et sur les premières sources écrites. Nous ne tiendrons toutefois pas compte des renseignements provenant de sources historiques tardives relatives aux communautés vivant au Mont Admirable aux $\mathrm{XI}^{\mathrm{e}}-\mathrm{XIII}^{\mathrm{e}}$ siècles et qui ne sont pas pertinentes pour la présente analyse ${ }^{5}$.

$$
* * *
$$

La vie de Syméon nous apprend que les principaux travaux de construction au Mont Admirable s'achevèrent en 551. C'est à cette date que furent consacrés l'église de la sainte Trinité (église centrale), le silo, l'octogone avec la colonne du Stylite ainsi que l'escalier monolithique conduisant au sommet, de la cuisine, de la boulangerie, de la forge et enfin du xenodochion ${ }^{6}$ (fig. 1) , $^{7}$, sachant que ces derniers n'étaient pas les premiers édifices érigés au sommet. Syméon et ses disciples auraient trouvé, sur l'emplacement du futur sanctuaire, des citernes creusées dans les flancs de la montagne (fig. 1) ${ }^{8}$. Après la mort de sainte Marthe, mère de Syméon, aux environs de 562, une basilique située au sud de l'église centrale fut élevée en mémoire de la sainte. D'après le récit de la vie de sainte Marthe, la construction aurait été achevée avant la mort de Syméon en $592^{9}$.

\footnotetext{
5 Des informations sur la vie des communautés monastiques vivant au Mont-Admirable aux X ${ }^{\mathrm{e}}$-XIII ${ }^{\mathrm{e}}$ siècles se trouvent dans: PeEters, «L'église Géorgienne du Clibanion au Mont Admirable »; CAHEN C. (1940), La Syrie du Nord à l'époque des croisades et la principauté franque d'Antioche, (Institut Français de Damas, Bibliothèque orientale, 1) Librairie orientaliste Paul Geuthner, Paris, p. 323-324; vAN DEN VEN, La vie ancienne de S. Syméon Stylite le Jeune (521-592), vol. I: Introduction et texte grec, p. $215^{*}-221^{*}$; Morray, «The Defences of the Monastery of St Simeon the Younger on Samandağ », p. 622-623.

6 VAn den Ven, La vie ancienne de S. Syméon Stylite le Jeune (521-592), vol. I: Introduction et texte grec, p. 99, 100, 113; cf. LAFONTAINE-Dosogne, Itinéraires archéologiques dans la région d'Antioche, p. 77-78; DJobadze, Archaeological Investigations, p. 58-59, 83.

7 La fig. 1 fut établie sur la base du plan de Wachtang Djobadze. Bien qu'il comporte des erreurs évidentes concernant le mur d'enceinte intérieur et extérieur, celles-ci n'ont cependant pas d'importance majeure pour l'évolution de l'architecture du site.

8 VAn Den Ven, La vie ancienne de S. Syméon Stylite le Jeune (521-592), vol. I: Introduction et texte grec, p. 97. On considère que la citerne située au nord de l'échapelle mortuaire nord-est et au sud de l'église Sainte-Marthe fut érigée avant la construction du sanctuaire chrétien: DJoBADze, Archaeological Investigations, p. 57, 94. Selon Wachtang Djobadze, à proximité de l'une de ces citernes se trouveraient des bâtiments de la caserne romaine: DJoBADZE, Archaeological Investigations, p. 57, 58.

9 VAN DEN VEN, «Le martyrium en triconque dans la vie de Sainte Marthe », p. 249-255; LAFOnTAINEDosogne, Itinéraires archéologiques dans la région d'Antioche, p. 125.
} 
- Période aven la construction du sanctuaire du monastère

- 551 après J.- C.

- 562-592 après J.- C.

- Période entre 592 après J.- C. et la construction du mur d'enceinte intérieur

- Période entre 592 après J.- C. et la construction du mur d'enceinte intérieur

- fin du VI's. et début du VII's. (?)

- Période entre 592 après J.- C. et la construction du mur d'enceinte intérieur

- Période entre 592 après J.- C. et la construction du mur d'enceinte extérieur

- Reconquête byzantine et $\mathrm{XI}^{*} \mathrm{~s}$.

- XII-XII's.

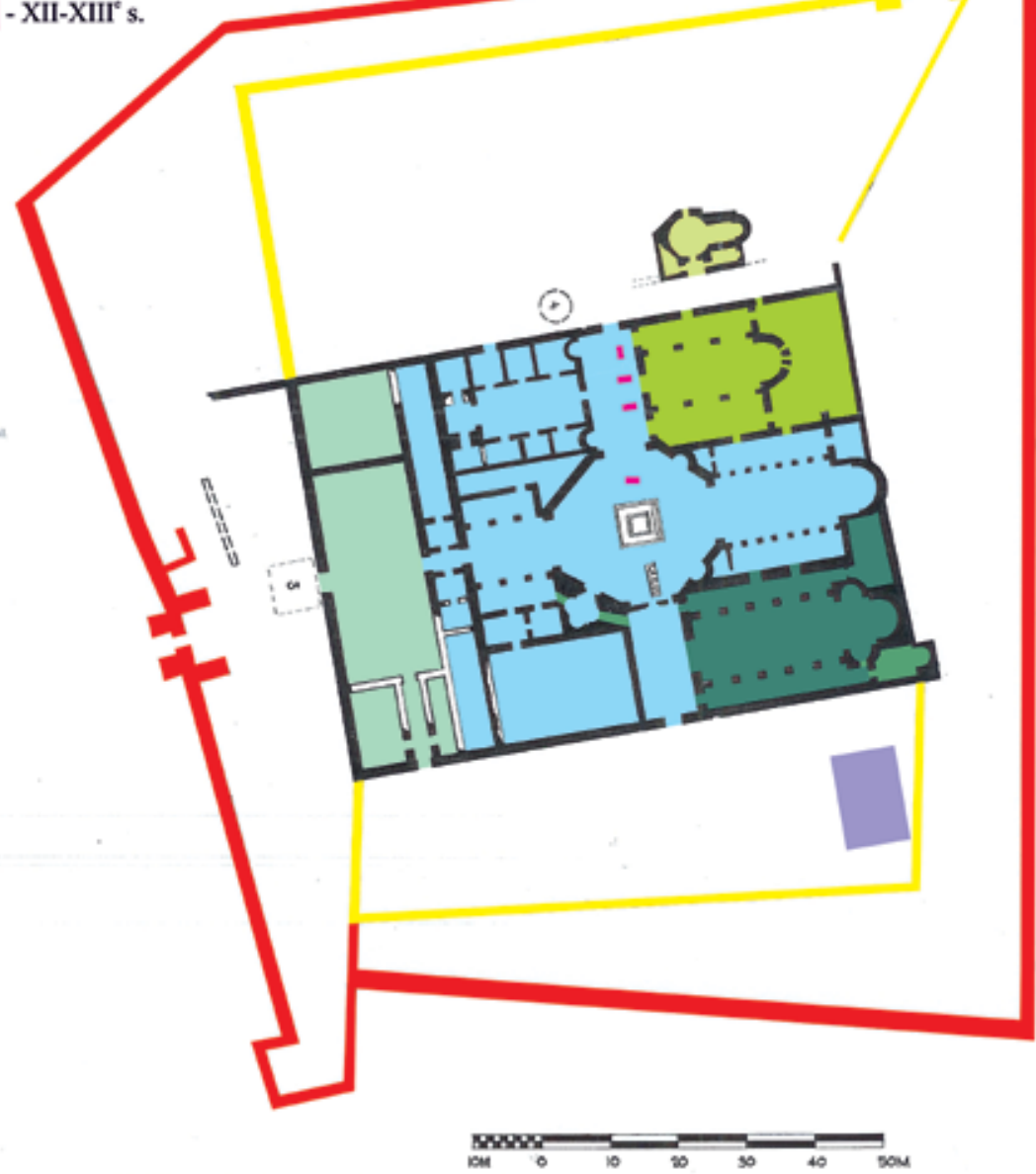

Fig. 1 - Plan du sanctuaire de Syméon-Stylite-le-Jeune avec indication des différentes étapes de la construction (M. Gwiazda d'après DJoBAZDE, Archaeological Investigations in the Region West of Antioch on-the-Orontes, plan F). 
Vient ensuite la construction de la porte nord-est du sanctuaire, et plus précisément de son entrée sud. Sur le linteau figure une inscription grecque au contenu incertain, qu'une analyse paléographique permet de dater $\mathrm{du} \mathrm{VI}^{\mathrm{e}}$ ou du début du vir ${ }^{\mathrm{e}}$ siècle, voire même des IX-X ${ }^{\mathrm{e}}$ siècles ${ }^{10}$. L'absence d'indications sur cette construction, dans les textes consacrés à la vie de Syméon et de sa mère, peut suggérer que l'édifice fut érigé après la mort du Stylite. La porte en question fait partie du mur intérieur du sanctuaire qui entourait les parties nord et sud de l'ensemble central. Une analyse des jonctions entre le mur et les restes de la construction, visibles sur le plan du sanctuaire, permet de constater que la chapelle sud de l'église sud (dédiée à sainte Marthe), ainsi que la basilique nord (probablement dédiée à Notre-Dame) et le parvis avec trois pièces attenantes, situées à l'ouest de l'atrium et des propylées, sont antérieurs ou contemporains au mur d'enceinte intérieur en question. En admettant (sur la base des informations acquises) que ces édifices soient antérieurs au mur d'enceinte, nous ne sommes pas en mesure de dire s'ils furent érigés à la même période, ou bien successivement, par exemple dans l'ordre suivant: chapelle, troisième église et cour supplémentaire. De même, nous ne sommes pas certains de la date de construction du couloir, qui traverse le tétraconque et qui relie l'atrium au couloir partant vers le sud de l'octogone ${ }^{11}$. Vraisemblablement, celui-là est contemporain ou postérieur à l'église Sainte-Marthe à laquelle il conduit indirectement.

Il en va de même pour le passage ouvert dans la partie sud du triconchos, vers l'église Sainte-Marthe ${ }^{12}$. Du point de vue fonctionnel, sa construction aurait eu un sens seulement après l'ajout de la chapelle au sud du triconchos. La présence, à la hauteur de l'entrée de la chapelle, de deux murs à appareils distincts peut être considérée comme preuve d'une construction ultérieure de la chapelle. Le mur sud de l'église, plus ancien, est composé de blocs de pierre de dimensions inégales, ce qui rend l'appareil visiblement irrégulier. En revanche, le mur sud de la chapelle fut érigé à partir de blocs allongés de même hauteur qui créent des rangées bien

\footnotetext{
${ }^{10}$ Le père Jean Mécérian considérait que l'inscription énumère les miracles du saint Syméon. Selon JeanPaul Rey Coquais, l'inscription mentionne la restauration du monastère survenue après la période du moine Angoulas, successeur de Syméon; voir Mécérian, «Les inscriptions du Mont Admirable », p. 316-321; ReY-CoquaIs, «Inscriptions du Mont Admirable», p. 204-214. Jacqueline Lafontaine-Dosogne opte pour une datation plus large de l'inscription: LAFONTAINE-DosogNE, Itinéraires archéologiques dans la région d'Antioche, p. 80. Frank Trombley, en se basant sur la police des caractères, la date du VI siècle: Trombley F.R. (2004), « Demographic and Cultural Transition in the Territorium of Antioch, $6^{\text {th }}-10^{\text {th }} \mathrm{c}$. », Topoi, Supplementum 5, p. 337-348.

${ }^{11}$ Cf. Duobadze, Archaeological Investigations, p. 72.

12 Ibid., p. 79.
} 
distinctes. De même, le mur nord de la chapelle en question n'a pas de jonctions avec les murs de l'église Sainte-Marthe ${ }^{13}$.

On peut en outre s'interroger sur la date de création du baptistère situé au nord de l'église nord. L'édifice fut daté par Wachtang Djobadze et par Bruno Dufay de la fin $\mathrm{du} \mathrm{VI}^{\mathrm{e}}$ ou du début du VII ${ }^{\mathrm{e}}$ siècle $^{14}$. Même si cette datation semble vraisemblable, elle manque de preuves convaincantes.

Les dernières réalisations significatives liées au développement du sanctuaire et du monastère de Syméon-Stylite-le-Jeune furent celles de la construction du mur d'enceinte extérieur et d'une chapelle mortuaire nord-est adjacente. Dans ce cas-là non plus, ni les sources écrites ni les sources archéologiques ne nous livrent d'informations univoques, qui permettraient d'indiquer avec précision la date de création de ces constructions. En outre, on n'a toujours pas réussi à élucider la relation chronologique entre ces édifices. Au vu d'un argumentaire très suggestif de Wachtang Djobadze, nous pouvons être certains seulement du fait que la chapelle mortuaire avec vestibule, ainsi que le mur d'enceinte extérieur furent ajoutés à la porte avec inscription déjà existante, elle-même faisant partie du mur d'enceinte intérieur. L'absence de jonction entre les murs du vestibule de la chapelle et les murs de la porte avec inscription confirme cette chronologie ${ }^{15}$.

Jacqueline Lafontaine-Dosogne a constaté que la création du mur autour du sanctuaire devrait être liée à la reconquête byzantine. Cette hypothèse se justifie du fait que le monastère de Syméon-Stylite-le-Grand à Qal'at Sem'an fut, à cette époque-là, entouré d'une muraille ${ }^{16}$. David Morray, après avoir analysé la construction et les finitions des pierres de taille utilisées pour la voûte de la majestueuse porte située dans la partie ouest du mur extérieur, suggère de dater sa construction de la fin du $\mathrm{XI}^{\mathrm{e}}$ siècle $^{17}$.

${ }^{13}$ Observation relative à l'absence de jonction entre les appareils des murs de la chapelle et ceux des murs de l'église faite par le présent auteur au moment de son séjour sur le site en 2008. L'absence de lien entre le mur nord de la chapelle et l'abside de l'église Sainte-Marthe est visible sur le plan de DJobADZE, Archaeological Investigations, plan G.

${ }^{14}$ Djobadze, Archaeological Investigations, p. 88; Dufay B. (1984), Immersions. Lieux et pratiques de l'initiation chrétienne dans le patriarcat d'Antioche (étude archéologique et liturgique des baptistères paléochrétiens), Paris (thèse de doctorat non publiée), p. 107. En essayant de déterminer la chronologie de la construction du baptistère, Wachtang Djobadze s'est basé sur les ressemblances de la technique utilisée dans les plus anciennes parties du sanctuaire. Les deux chercheurs soulignent également l'absence d'informations sur le baptistère dans les vies de saint Syméon et de sainte Marthe, ce qui permet de croire qu'il fut érigé après la mort du Stylite.

15 DJobadze, Archaeological Investigations, p. 89.

${ }^{16}$ Lafontaine-Dosogne, Itinéraires archéologiques dans la région d'Antioche, p. 75-76; Wachtang Djobadze est plus prudent sur ce sujet, datant l'élévation du mur d'enceinte extérieur de la période entre la fin du $\mathrm{X}^{\mathrm{e}}$ et la moitié du XIII ${ }^{\mathrm{e}}$ siècle. DJobadZE, Archaeological Investigations, p. 90.

${ }^{17}$ Morray, «The Defences of the Monastery of St Simeon the Younger on Samandağ », p. 620, 622, 
Les éléments les plus récents du sanctuaire sont des tombeaux creusés dans la roche, situés dans le corridor à l'ouest de l'église nord et à proximité de la colonne du Stylite dans l'octogone. Deux des sépultures étaient couvertes de tables aux épitaphes mentionnant deux dates annuelles: 1193 et 1266. Ces inscriptions représentent les derniers objets datés avec précision, trouvés sur le site de fouilles ${ }^{18}$. Nous n'avons aucune information sur le fonctionnement du monastère relative à la période postérieure à 1266 provenant des récits historiques. C'est pour cette raison-là qu'on suppose qu'il fut détruit, en même temps qu'Antioche, suite à l'invasion du sultan Baybars en $1268^{19}$.

Afin de compléter cette présentation de l'évolution du sanctuaire, nous nous servirons de la documentation photographique établie lors des fouilles du père Jean Mécérian. Dans les passages situés dans différentes parties du sanctuaire, nous découvrons une série de bouchages, représentant les éléments les plus intéressants ${ }^{20}$. Le premier d'entre eux se situait dans le passage entre l'octogone et le tétraconque partiellement creusés dans la roche (fig. 2). Son parement correspondait au parement des murs de l'octogone. Le bouchage est constitué de blocs de pierre de longueurs inégales, taillés de manière régulière, scellées vraisemblablement par le mortier. Sur la photo on voit quatre rangées de pierre conservées. Dans le cas des deux rangées inférieures, les pierres étaient assorties en fonction de leur hauteur. Des deux rangées supérieures, il ne restait plus qu'un seul bloc.

Entre les socles, qui flanquent l'entrée du tétraconque, on aperçoit trois rangées de pierres qui, vraisemblablement, composaient le parement d'une plate-forme précédant le bouchage situé derrière elle. La construction fut réalisée selon une technique proche de celle utilisée pour le bouchage décrit plus haut, avec des pierres de longueurs inégales, disposées en trois rangées de hauteur rapprochée. En outre, sur le socle droit, qui sépare les tables de l'octogone collées contre le mur de l'entrée du

${ }^{18}$ MécÉrian, «Les inscriptions du Mont Admirable », p. 321-324; ReY-CoQUaIs, « Inscriptions du Mont Admirable », p. 219-223.

${ }^{19}$ VAN DEN Ven, La vie ancienne de S. Syméon Stylite le Jeune (521-592), vol. I: Introduction et texte grec, p. 220*-221*; DJobadze, Archaeological Investigations, p. 97-98; Morray, « The Defences of the Monastery of St Simeon the Younger on Samandağ », p. 623.

${ }^{20}$ Dans 1'enclos du sanctuaire, Wachtang Djobadze a également indiqué des bouchages dans 1'un des passages reliant l'église centrale et l'église sud, dans deux des trois fenêtres situées dans l'abside de l'église nord, dans le mur ouest de l'hospice (xenodochion) ainsi que dans l'un des murs du baptistère: DJOBADZE, Archaeological Investigations, p. 75, 82, 87. 


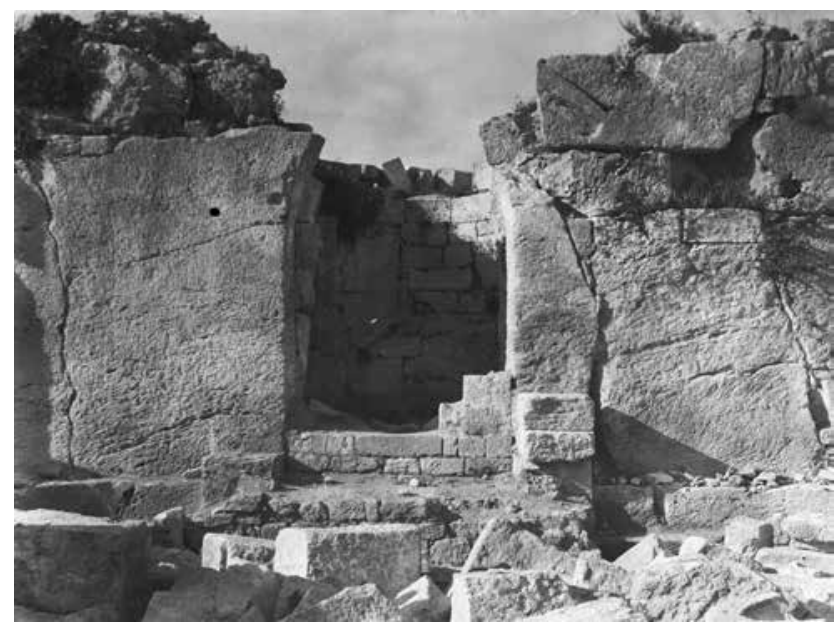

Fig. 2 - L'entrée nord-est du tétraconque avec des restes de son bouchage (enveloppe 23N PCMA).

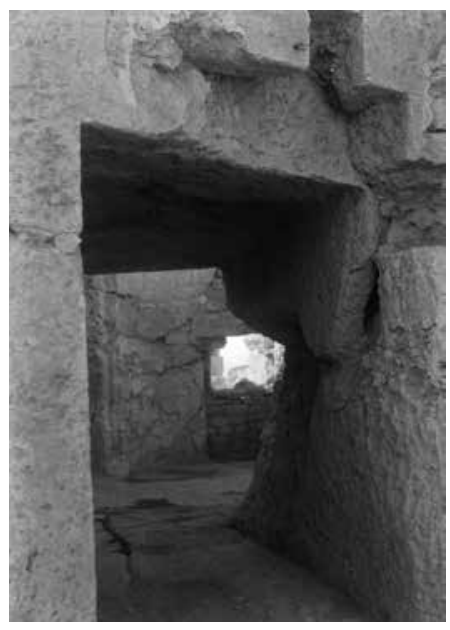

Fig. 3 - Le bouchage du couloir nord-ouest du tétraconque, vu du sud-est (enveloppe 23N PCMA).

tétraconque, se trouvent deux autres blocs de pierre. Il est difficile d'affirmer qu'ils firent partie de la construction disparue.

Une autre photo du lot représente un passage qui mène, par le tétraconque, du nord-ouest au sud-est et relie indirectement l'atrium et le vestibule de l'église sud (fig. 3). Au deuxième plan, nous pouvons remarquer une entrée du côté de l'atrium. S'y trouve un bouchage conservé jusqu'à mi-hauteur de l'entrée. Nous y apercevons une construction partiellement irrégulière, réalisée en blocs de pierre posés en rangées de hauteurs inégales. Sur une photo prise dans l'atrium (fig. 4) apparaît le même couloir avec un bouchage dans l'entrée du côté nord-ouest. Nous pouvons en conclure qu'il occupait toute la longueur nord-ouest du passage du tetrachonchos.

Un autre bouchage se trouve dans un passage entre la nef nord et une pièce annexe de l'église centrale (fig. 5) ${ }^{21}$. Il constitue un plateau, érigé de quelques blocs de pierre et de gravats aux formes irrégulières, qui atteint presque la mi-hauteur de l'entrée, en correspondance avec la deuxième rangée (en comptant du bas) de blocs de pierre du mur de cette partie de l'église. Par ailleurs, les pierres de deux autres rangées, jouant aussi le rôle d'embrasures de la première entrée, furent ultérieurement légèrement coupées et privées de jambages. Ainsi fut créée une « fenêtre » de forme quasi carrée. Un autre élément attire l'attention, à savoir une coquille (concha) du linteau. Aucun

\footnotetext{
${ }^{21}$ Ce bouchage est également visible sur une photo publiée par Wachtang Djobadze, cependant ce dernier ne le commente pas et ne le fait figurer sur aucun des plans: DJobadzE, Archaeological Investigations, fig. 129.
} 


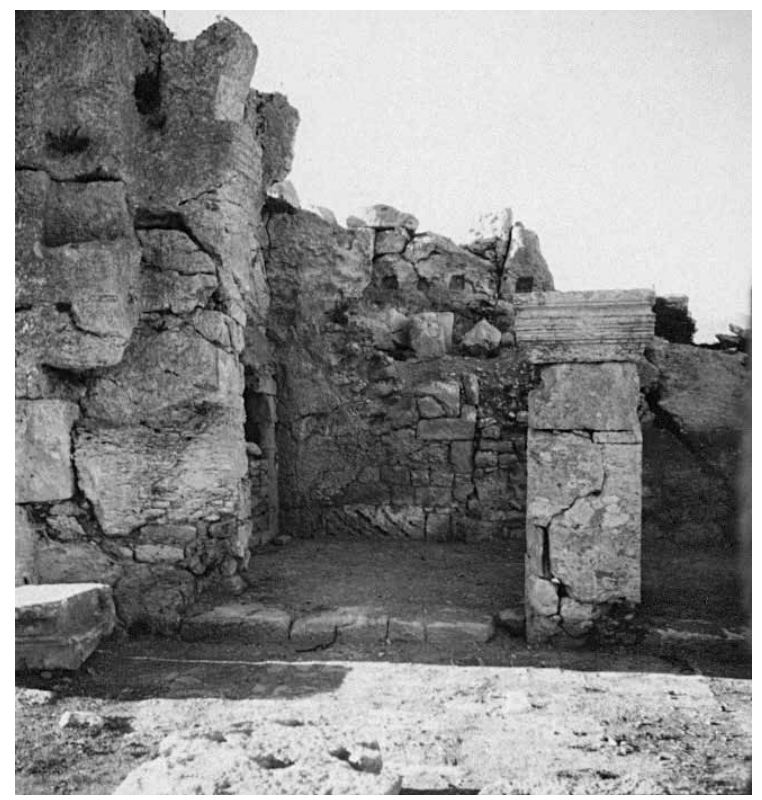

Fig. 4 - Vue, du côté de l'atrium, sur le bouchage dans le couloir nord-ouest du tétraconque (enveloppe 49 PCMA).

autre linteau du site n'a d'ornement de ce type. Sur la photo qui montre le même passage, mais du côté est, on voit le parement du bouchage qui correspond dans son intégralité au parement du mur, et, aux extrémités du linteau, se trouvent des trous qui pouvaient servir au montage d'une tringle à rideaux pour séparer l'intérieur de la pièce (fig. 6). Tous ces éléments (planche, jambages coupés, coquille et trous permettant l'installation du tissu) peuvent indiquer que le passage a pu être utilisé comme une niche dont la fonction reste cependant difficile à déterminer. Elle pouvait servir à l'exposition de reliques et d'un tableau sacré22.

En outre, à une période indéterminée, dans un passage entre la nef sud de l'église nord et l'arrière de l'église situé dans la partie est de l'abside, apparut une nouvelle construction sous forme d'un bouchage, qui remplit environ deux tiers de la hauteur de l'embrasure de la porte (à droite fig. 6) ${ }^{23}$.

Aussi bien sur le plan du père Jean Mécérian que sur les plans de Wachtang Djobadze, on voit dans le mur nord de l'église nord, une porte qui mène vers le baptistère. Sur la base d'une des photos d'archives du père Jean Mécérian, nous pouvons

\footnotetext{
${ }^{22}$ Wachtang Djobadze considère que le tableau ou les reliques se trouvaient dans une niche de forme similaire, creusée dans le couloir sud partant de l'octogone: DJobadze, Archaeological Investigations, p. 83, 96, fig. 201.

${ }^{23}$ Ce bouchage est visible sur une photo publiée par Wachtang Djobadze. De même que dans le cas précédent, il n'a pas été décrit et ne figure pas sur les plans: Duobadze, Archaeological Investigations, fig. 195.
} 




Fig. 5 - Vue, du côté ouest, sur le bouchage dans le passage entre la nef nord et une pièce annexe de l'église centrale (enveloppe 49 PCMA).



Fig. 6 - Vue, du côté ouest, sur le bouchage du passage entre la nef nord et une pièce annexe de l'église centrale et sur le bouchage du passage entre la nef sud et l'arrière de l'église nord (enveloppe 44 PCMA).

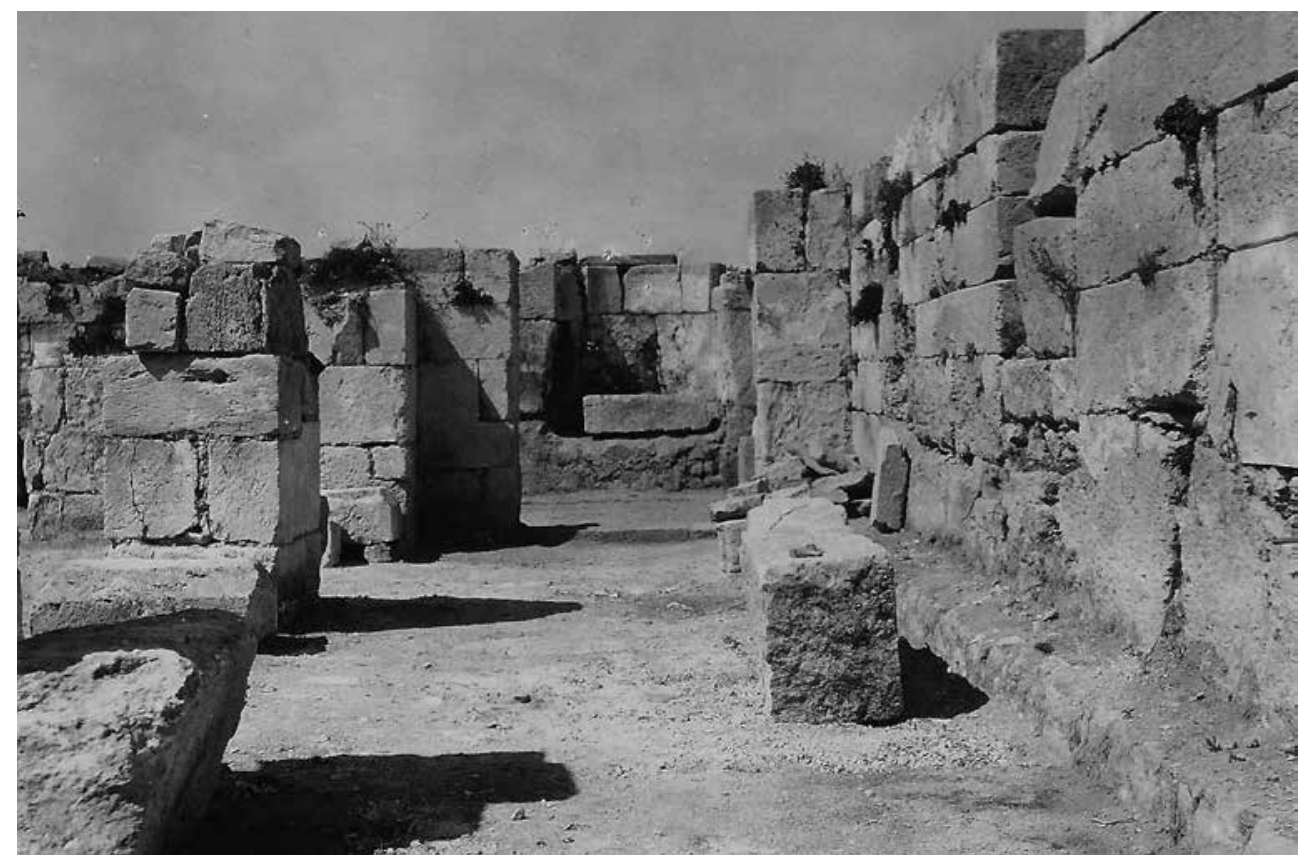

Fig. 7 - Vue, du côté est, sur la nef nord et sur le mur nord avec l'entrée bouchée à l'église nord (enveloppe 2.4-3 PCMA). 


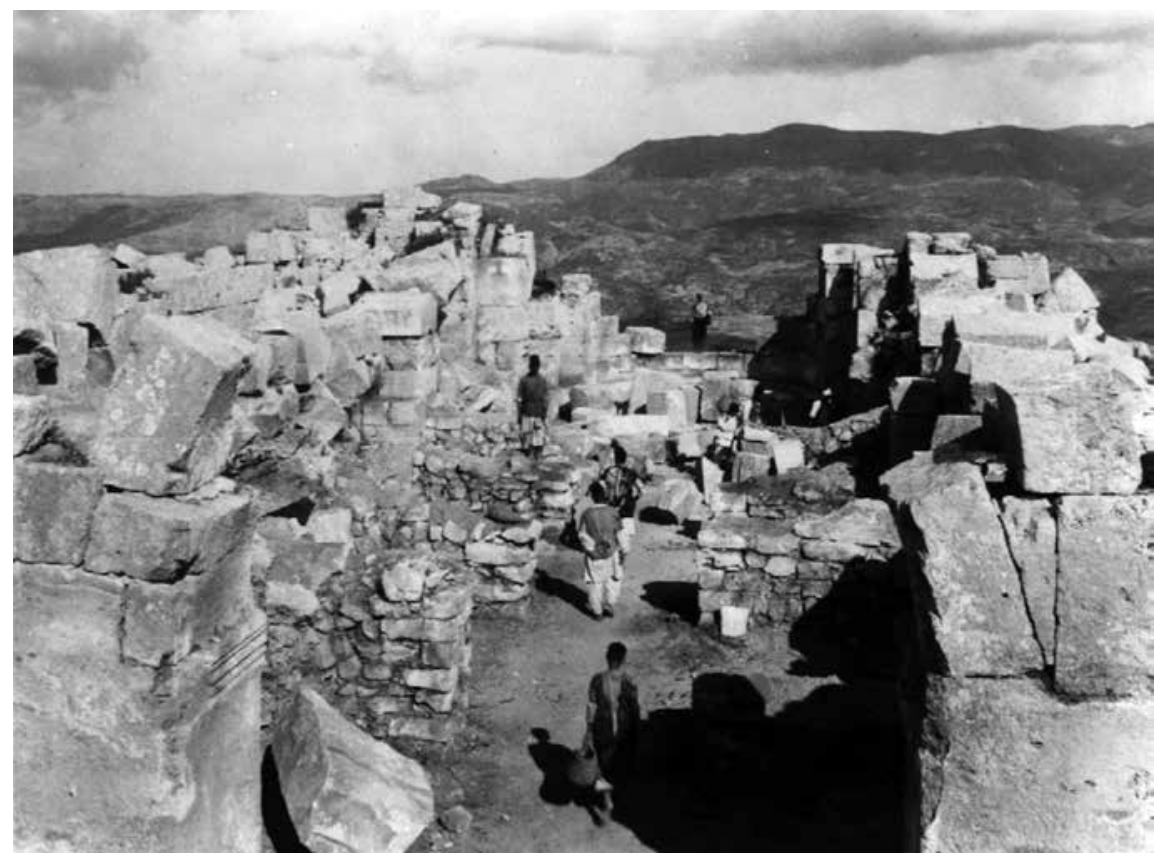

Fig. 8 - La nef principale de l'église sud vue à partir de l'ouest (enveloppe 3 PCMA).

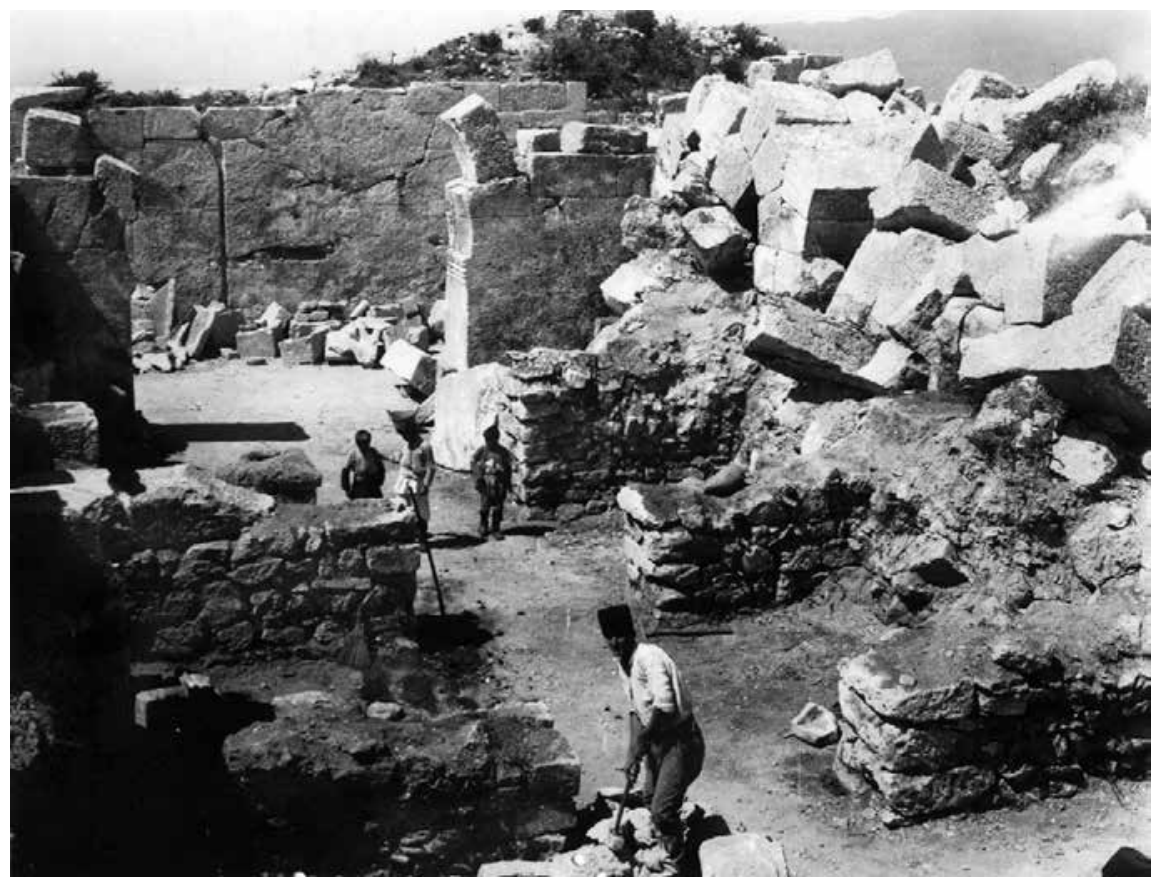

Fig. 9 - Vue sur la nef principale de l'église sud à partir du sud-est (enveloppe 95 PCMA). 
constater qu'à un moment indéterminé, ce passage fut entièrement bloqué à l'aide de blocs de pierre (à droite on peut voir une partie de ce bouchage fig. 7).

$\mathrm{Au}$ cours des fouilles menées par le père Jean Mécérian, on a retrouvé, dans la nef centrale de l'église sud, quatre paires de rangées de murs perpendiculaires à l'axe de la basilique (fig. 8 et 9). Ils sont attenants aux piliers sur lesquels reposaient les arcades qui soutenaient la construction du toit. Ils furent érigés à partir de petits blocs de pierre et de gravats. La hauteur conservée, en prenant en considération l'échelle humaine, se situe entre $0,5 \mathrm{~m}$ et $2 \mathrm{~m}$ environ. La distance entre différentes paires de murs pouvait aller de 2 à 2,5 m environ. Aucune photo d'archives déposées à la Bibliothèque Orientale de 1'Université Saint-Joseph ne laisse voir si les murs avaient leur prolongement dans les nefs latérales de la basilique. Afin de déterminer la fonction de ces éléments, nous devons avoir recours aux analogies avec d'autres sites archéologiques. À la basilique Sainte-Croix à Resafa, entre les arcades de la nef centrale, on érigea des arcs supplémentaires. Dans certains cas, l'espace entre eux fut emmuré. De pareils procédés visaient à renforcer l'architecture de l'église, qui avait subi des dommages causés par le tremblement de terre ${ }^{24}$. Une autre analogie, relative aux éléments en question, nous renvoie à l'église ouest d'Alahan. Après une catastrophe peu connue, cette basilique à trois nefs, fut complètement reconstruite. Une partie des colonnes de la nef centrale fut enlevée et remplacée par des murs continus auxquels, à distance égale, on ajouta des piliers perpendiculaires à l'axe de l'édifice. Michael Gough suggère qu'il s'agissait d'éléments liés à la toiture de la nef de l'église reconstruite ${ }^{25}$. Il n'est pas à exclure que les murs transversaux du Mont Admirable aient rempli la même fonction. Il est possible qu'à la suite d'un tremblement de terre, il fut nécessaire de renforcer les piliers à l'aide des arcades qui pourraient séparer les nefs, ou peut-être même il fallut poser une structure pour soutenir le toit.

Devant l'entrée sud donnant sur le couloir sud qui partait de l'octogone, se trouvaient trois autres éléments non mentionnés dans les rapports de fouilles. Le premier est le dallage en pierre (fig. 10), lequel une fois retiré, dévoila l'existence d'un escalier (fig. 11). À l'est de l'escalier, sur le parement extérieur du mur sud de l'église sud, on aperçoit aussi une niche creusée dans une roche solide.

\footnotetext{
${ }^{24}$ Ulbert T. (1986), Die Basilika des Heiligen Kreuzes in Resafa-Sergiupolis, (Resafa, 2) Philipp von Zabern, Mainz am Rhein, annexes 9 et 10.

${ }^{25}$ Gough M. (1962), « The Church of the Evangelists at Alahan: A Preliminary Report », Anatolian Studies 12, p. 176, 177; id. (1963), «Excavations at Alahan Monastery: Second Preliminary Report », Anatolian Studies 13, p. 106, fig. 1, pl. XXX, b; id. (1968), «Alahan Monastery: Fifth Preliminary Report», Anatolian Studies 18, p. 159, 160.
} 


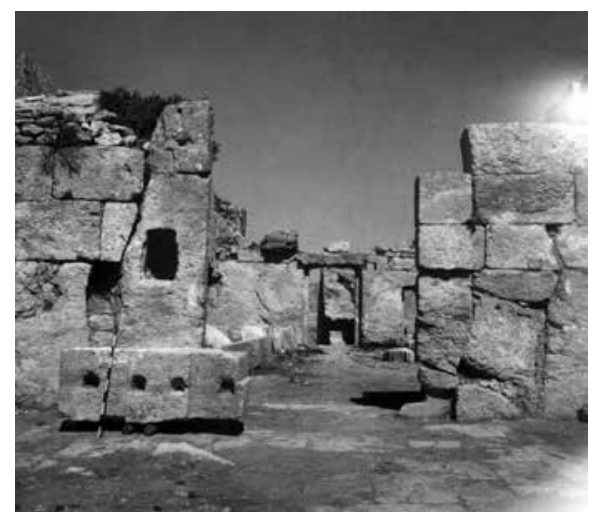

Fig. 10 - L'entrée sud vers la partie centrale du monastère. Vue prise du sud (enveloppe 49 PCMA).

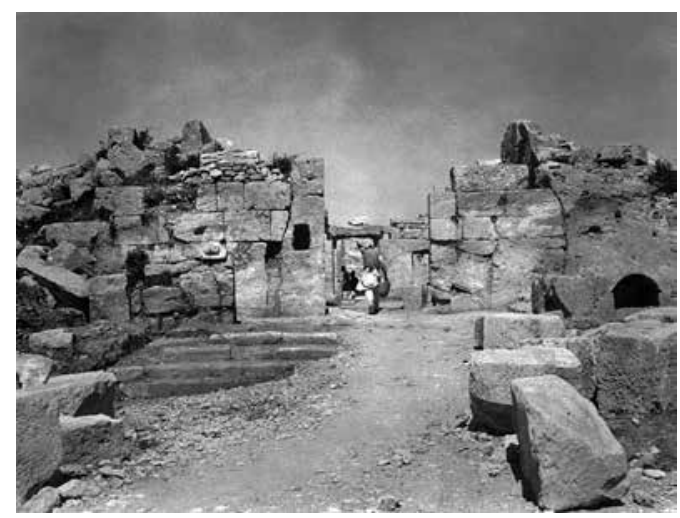

Fig. 11 - L'entrée sud vers la partie centrale du monastère. Vue prise du sud (enveloppe 48 PCMA).

Il est nécessaire de compléter les informations relatives au mur est de l'ensemble central. Sur le plan de Wachtang Djobadze, les appareils des murs est des pièces annexes nord de l'église sud, ainsi que ceux de l'église centrale sont reliés aux murs des absides de ces mêmes églises ${ }^{26}$. Cependant, les photos du père Jean Mécérian laissent plutôt conclure que ces murs n'étaient pas reliés aux absides des églises mentionnées plus haut. Ils ne faisaient qu'y attenir, en ayant des appareils de maçonnerie distincts, ajoutés à une période difficile à déterminer (fig. 12 et 13). Sur une des photos (fig. 12), on voit aussi un fragment du balcon (?) qui dépasse le revêtement du mur. Il ne figure sur aucun plan existant du site ${ }^{27}$.

Un autre élément intéressant, qu'on retrouve sur les photos du père Jean Mécérian, est la porte dite d'Antioche, qui constitue un ensemble d'édifices situé dans la partie nord-est du sanctuaire. Cet ensemble est composé de la porte centrale intérieure avec une inscription sur la façade nord du linteau, ainsi que d'une chapelle mortuaire avec vestibule et d'une porte extérieure du côté nord et d'un mur d'enceinte extérieur. Pour cette partie de l'enclos, il est très difficile d'indiquer avec précision les différentes phases de la construction et leurs relations les unes aux autres. Néanmoins, grâce aux photos du père Jean Mécérian, on peut distinguer des éléments suivants :

\footnotetext{
${ }^{26}$ DJobadze, Archaeological Investigations, plan G.

${ }^{27}$ Sa présence fut mentionnée par LAFOnTAINE-Dosogne, Itinéraires archéologiques dans la région d'Antioche, p. 148, fig. 69.
} 


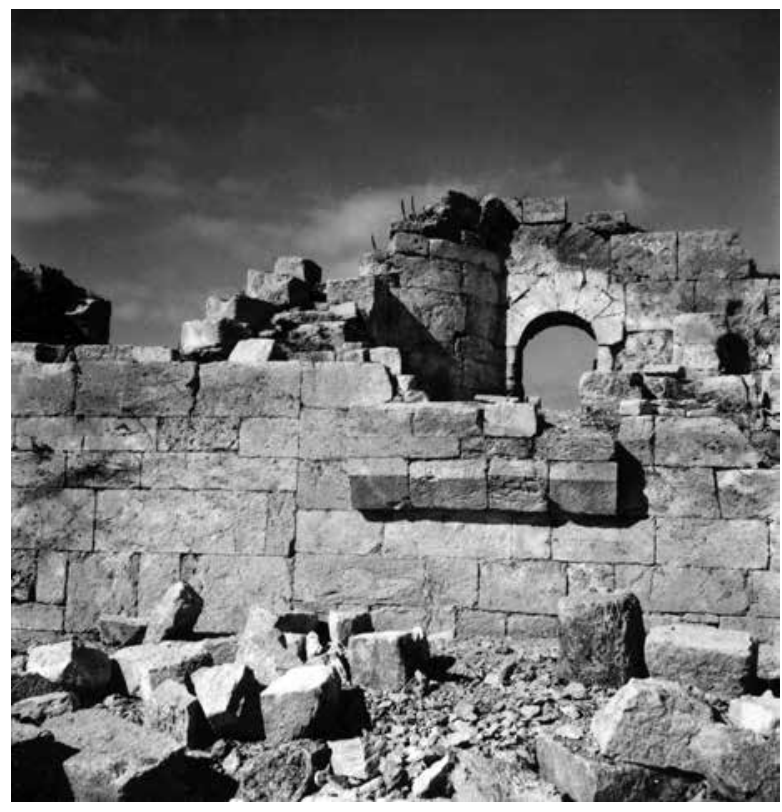

Fig. 12 - Vue du côté est sur la partie sud du mur est de l'ensemble central (enveloppe 49 PCMA).

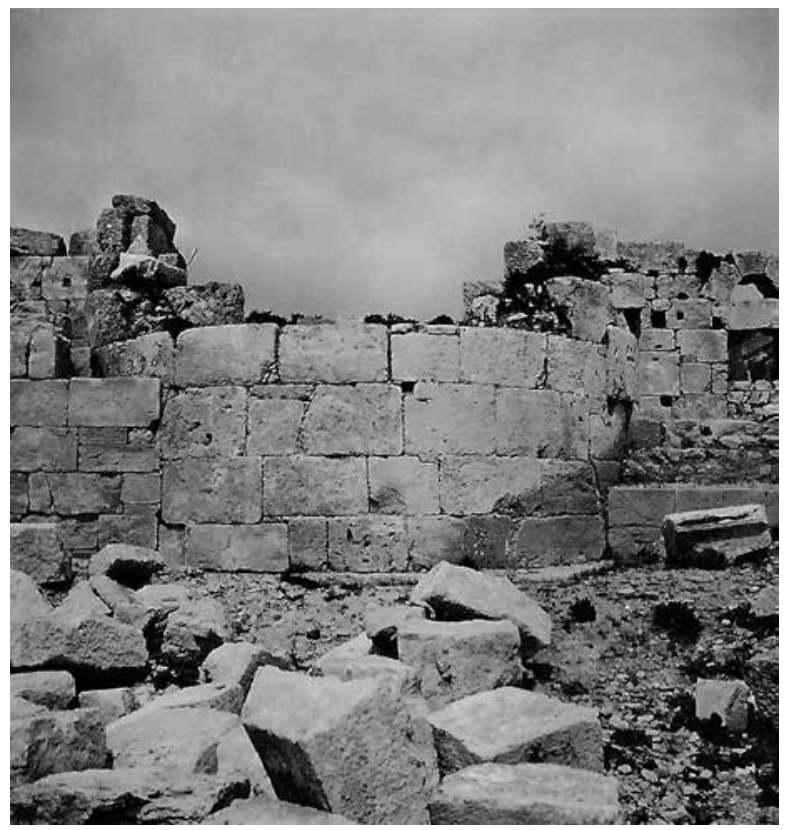

Fig. 13 - Vue du côté est sur l'abside de l'église centrale (enveloppe 2.4-3 PCMA). 


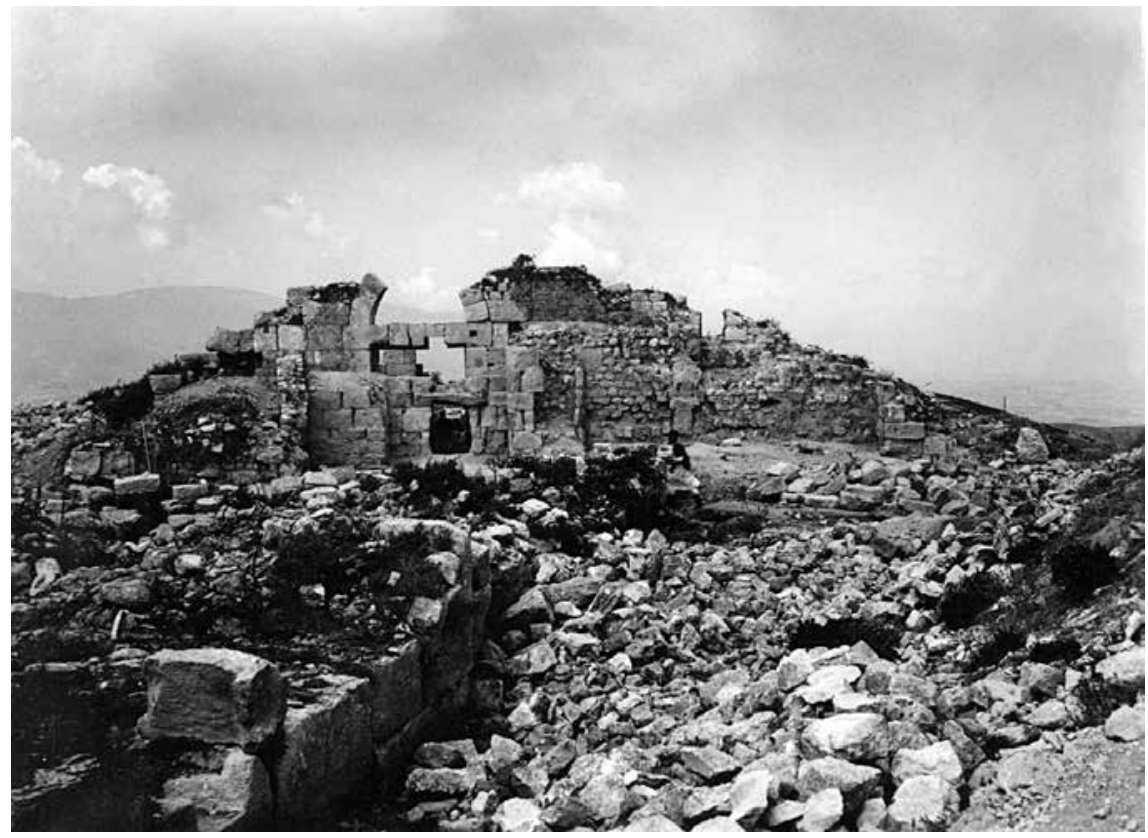

Fig. 14 - Vue sur la porte d'Antioche du côté sud (enveloppe 11 PCMA).



Fig. 15 - Vue sur la porte d'Antioche du côté sud (enveloppe 45 PCMA). 
- le mur attenant à la façade sud de la porte sud (fig. 14), avec une petite embrasure permettant le passage de l'autre côté de la porte. Dans la partie ouest, le mur est soit plus épais, soit doublé d'un autre mur ${ }^{28}$.

- Le mur perpendiculaire à la façade sud de la partie ouest du mur de la porte sud (fig. 14 et 15, partie gauche), érigé à partir de petits blocs de pierre et de gravats. Sur les photos, il est impossible de voir s'il est relié aux appareils du mur de la porte sud, ce qui pourrait suggérer qu'il est antérieur à la porte. Du côté est, son parement était couvert d'enduit et de peintures très mal conservées ${ }^{29}$.

- Le mur sud de la chapelle érigé de petits blocs de pierre (fig. 15 en haut à droite), constituant le prolongement de la partie est du mur de la porte sud.

- Le mur attenant au mur sud de la chapelle, érigé de petits blocs de pierre posés en rangées de hauteurs irrégulières (fig. 14 et 15).

- L'avant-corps attenant au mur sud de la porte sud et au mur sud de la chapelle, probablement relié au mur de la porte, même si la relation avec les murs de la chapelle n'est pas très claire.

- Une niche rectangulaire est aménagée dans la partie ouest de la façade sud de la porte sud (fig. 15). À l'intérieur, se trouvait une peinture représentant la croix latine, dont la branche inférieure était habillée d'un rameau alors que les extrémités des bras supérieurs étaient ramifiées et ornées d'anneaux. Entre les bras de la croix se trouvait une inscription. L'ensemble était entouré d'un cadre rectangulaire. Tous ces éléments furent exécutés avec une peinture foncée sur un fond clair (fig. 16).

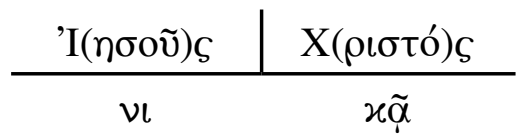

Des formules de ce type avaient un caractère apotropaïque, ce qui en l'occurrence est confirmé par l'emplacement de la peinture sur la porte d'entrée ${ }^{30}$. De telles ins-

${ }^{28}$ Ce rétrécissement de la porte est marqué sur un plan publié par Mécérian en 1936, ainsi que sur la photo de l'inscription du linteau de la porte: MécérIAN, «Les inscriptions du Mont Admirable », pl. 7.3.

${ }^{29}$ Les photos représentant ces peintures se trouvent dans les enveloppes aux numéros suivants: 5N PCMA, 8N PCMA, 44 PCMA.

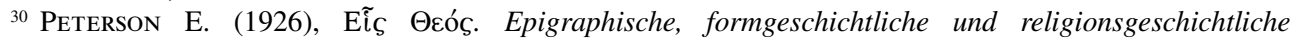
Untersuchungen, (Forschungen zur Religion und Literatur des Alten und Neuen Testaments, 24) Vandenhoeck \& Ruprecht, Göttingen, p. 157, 161. Cf. Inscriptions de ce type du territoire de SyroPalestine: Prentice W.K. (1908), Greek and Latin Inscriptions, Part III of the Publications of an American Archaeological Expedition to Syria 1899-1900, Century Co., New York, $\mathrm{n}^{\circ}$ 124, 201, 210, 219, 234, 237 , 255 ; et notamment: Piccirillo M. (1989), Chiese e Mosaici di Madaba, (Publications of the Studium Biblicum Franciscanum, Collectio maior, 34) Franciscan Printing Press, Jerusalem, p. 118; ZaYAdine F. (1994), «Ayla-'Aqaba in the Light of Recent Excavations », Annual of the Department of Antiquities 
criptions placées au cœur de la croix apparaissent souvent sur les pièces de monnaie byzantines et sur les monnaies croisées des $\mathrm{XI}^{\mathrm{e}}$ et $\mathrm{XII}^{\mathrm{e}}$ siècles. Malgré certaines différences iconographiques (telles que par exemple la croix grecque à la place de la croix latine sur les monnaies de Tancrède et l'absence de rameau sur la plupart des monnaies byzantines), on peut supposer qu'aussi bien dans le monnayage que dans le cas de la peinture en question, ce motif avait une même origine ${ }^{31}$.

- Le bouchage de la porte nord réalisé en deux appareils distincts. Du côté nord, le bouchage fut réalisé à partir de grands blocs de pierre de taille régulière et posés en rangées (fig. 17), reposant directement sur le seuil relativement haut par rapport au niveau de la circulation du côté extérieur de la porte. Cette importante différence de niveaux, avant la fermeture de ce passage à la circulation, fut probablement nivelée à l'aide d'un escalier en bois, dont l'existence est rappelée par deux trous dans le seuil. Le bouchage du côté sud fut réalisé à partir de petits blocs de pierre (fig. 15). Les parements des deux parties du bouchage correspondent aux parements extérieur et intérieur des murs de la porte nord.

Une photo aérienne verticale du sanctuaire prise au moment des fouilles dans les années 1930 donne une nouvelle perspective aux plans du site publiés. En superposant cette photo aérienne, les plans du père Jean Mécérian (traits blancs) et ceux de Wachtang Djobadze (plan d'ensemble - traits gris; plan de l'ensemble central traits noirs), on peut constater certaines inexactitudes (fig. 18) ${ }^{32}$ :

- En dépit du fait que le plan de l'ensemble central élaboré par Wachtang Djobadze correspond parfaitement à la photo, le plan global du sanctuaire comprenant les murs d'enceinte doit être considéré pour le moins comme peu précis. Pour cette partie-là, le plan de Jean Mécérian est plus précis, sans être dépourvu d'erreurs. La porte ouest et la corne sud-ouest du mur extérieur ont été déplacées de quelques mètres vers le sud, et leur contour est légèrement déformé. Leur juste emplacement est indiqué sur la photo par un trait noir et blanc interrompu.

of Jordan 38, p. 489, 494, fig. 8, 9. Au sujet de l'évolution et la signification de ce type d'inscription: Frolow A. (1956), «IC XC NIKA », Byzantinoslavica 17/1, p. 98-113.

${ }^{31}$ Morrisson C. (1970), Catalogue des monnaies byzantines de la Bibliothèque Nationale, 2 vol., vol. II: De Philippicus a Alexis III (711-1204), Bibliothèque Nationale, Paris, p. 600 (des folles anonymes de classe C); Metcalf D.M. (1995), Coinage of the Crusades and the Latin East in the Ashmolean Museum, Oxford, $2^{\text {nd }}$ ed., (Special Publications - Royal Numismatic Society, 28) Royal Numismatic Society - Society for the Study of the Crusades and the Latin East, Londres, p. 63-70 (Tancrède); Hendy M.F. (1999), Catalogue of the Byzantine Coins in the Dumbarton Oaks Collection and in the Whittemore Collection, vol. IV: Alexius I to Michael VIII: 1081-1261, Dumbarton Oaks Research Library and Collection, Washington (D.C.), p. 213-214 (Alexis I ${ }^{\text {er }}$ Comnène), 429 (Trabzon).

${ }^{32}$ Millet, «Rapport du P. Mécérian sur les fouilles au monastère de Saint-Syméon le Jeune au Mont Admirable (Syrie) »; DJobaDZE, Archaeological Investigations, plan F et G. 


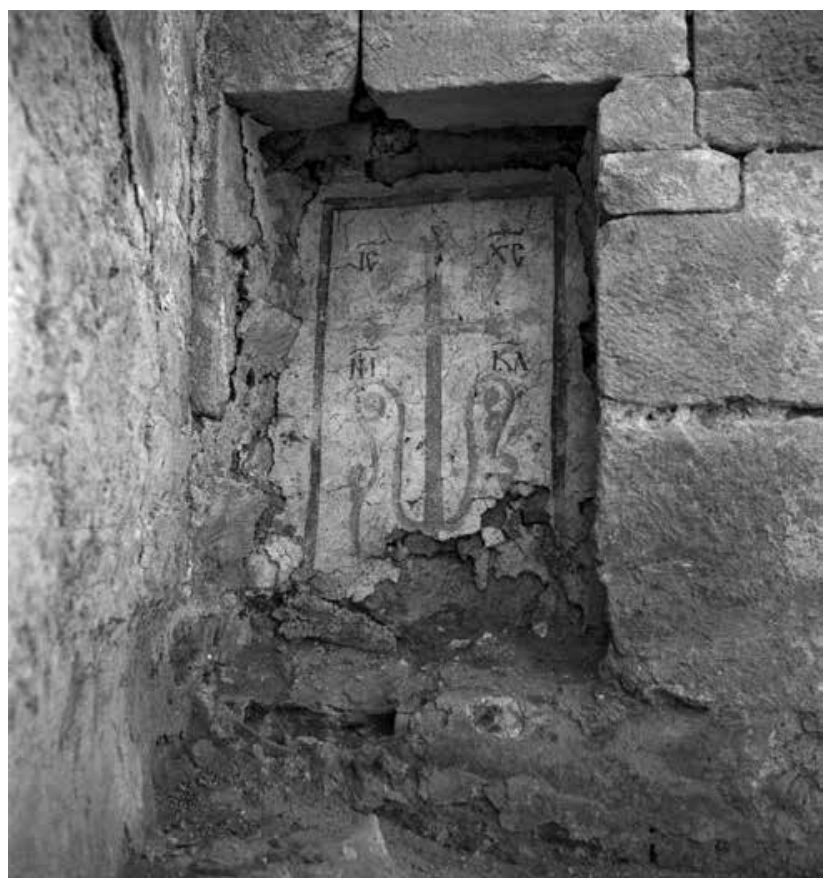

Fig. 16 - Croix peinte avec inscription dans une niche aménagée dans le mur sud de la porte d'Antioche (enveloppe 6N PCMA).

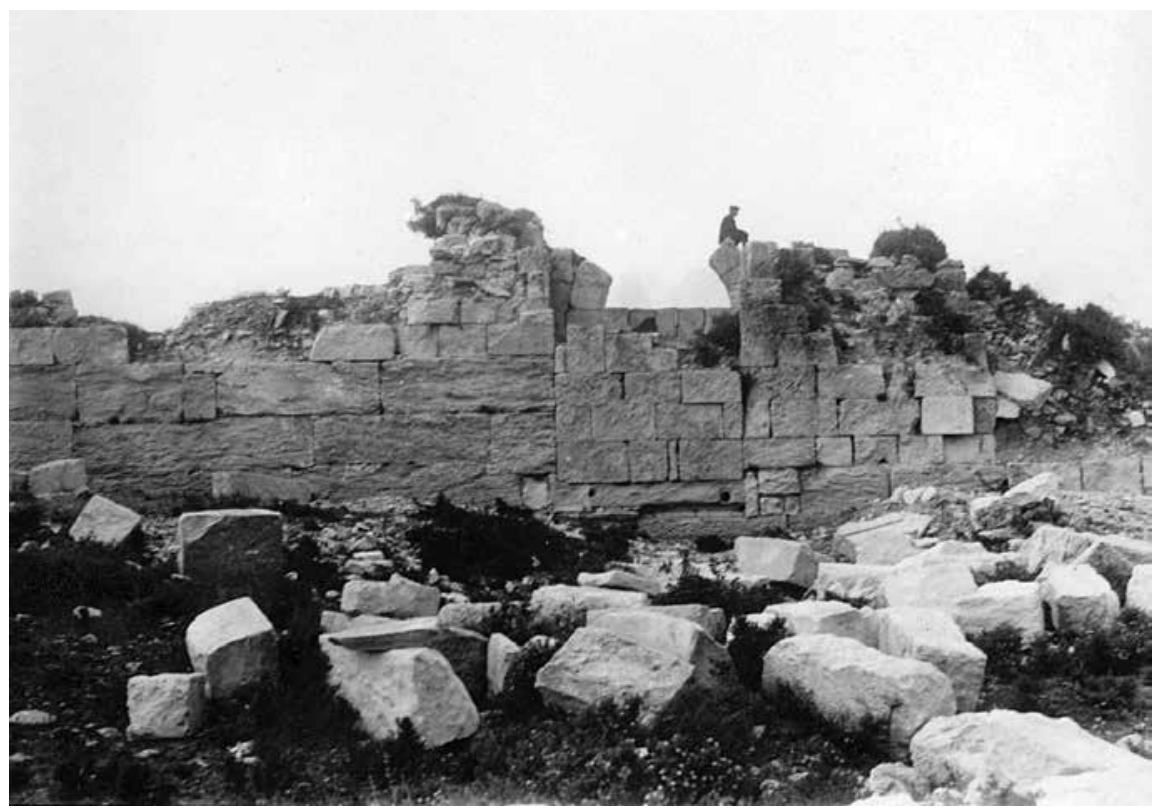

Fig. 17 - Vue sur la porte d'Antioche du côté nord (45 PCMA). 


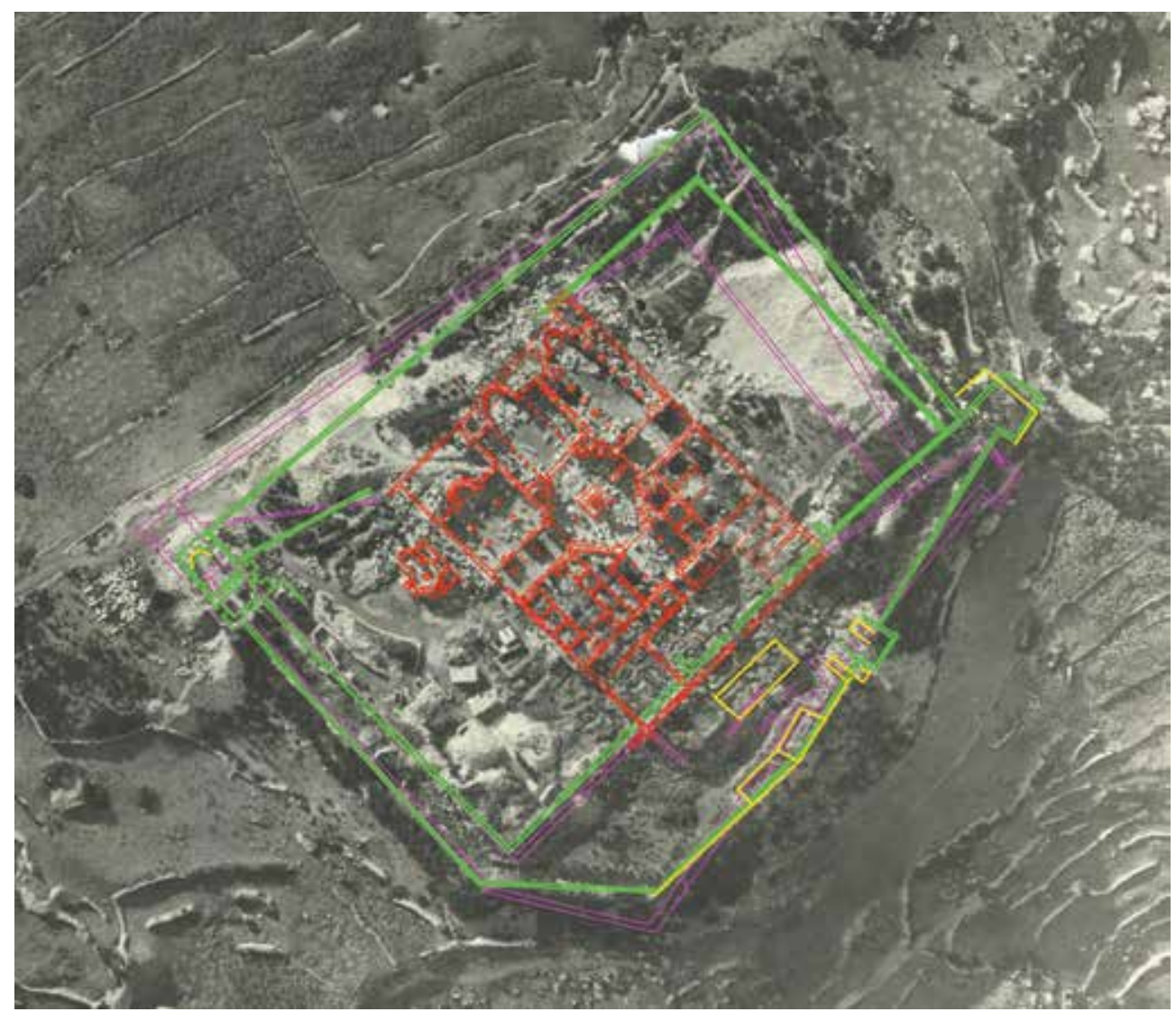

Fig. 18 - Photo aérienne du sanctuaire de Saint-Syméon-Stylite-le-Jeune (enveloppe 97 PCMA) avec superposition des plans réalisés par Jean Mécérian (MiLlET, « Rapport du P. Mécérian sur les fouilles au monastère de Saint-Syméon le Jeune au Mont Admirable (Syrie) », et DJobadze, Archaeological Investigations in the Region West of Antioch on-the-Orontes). 


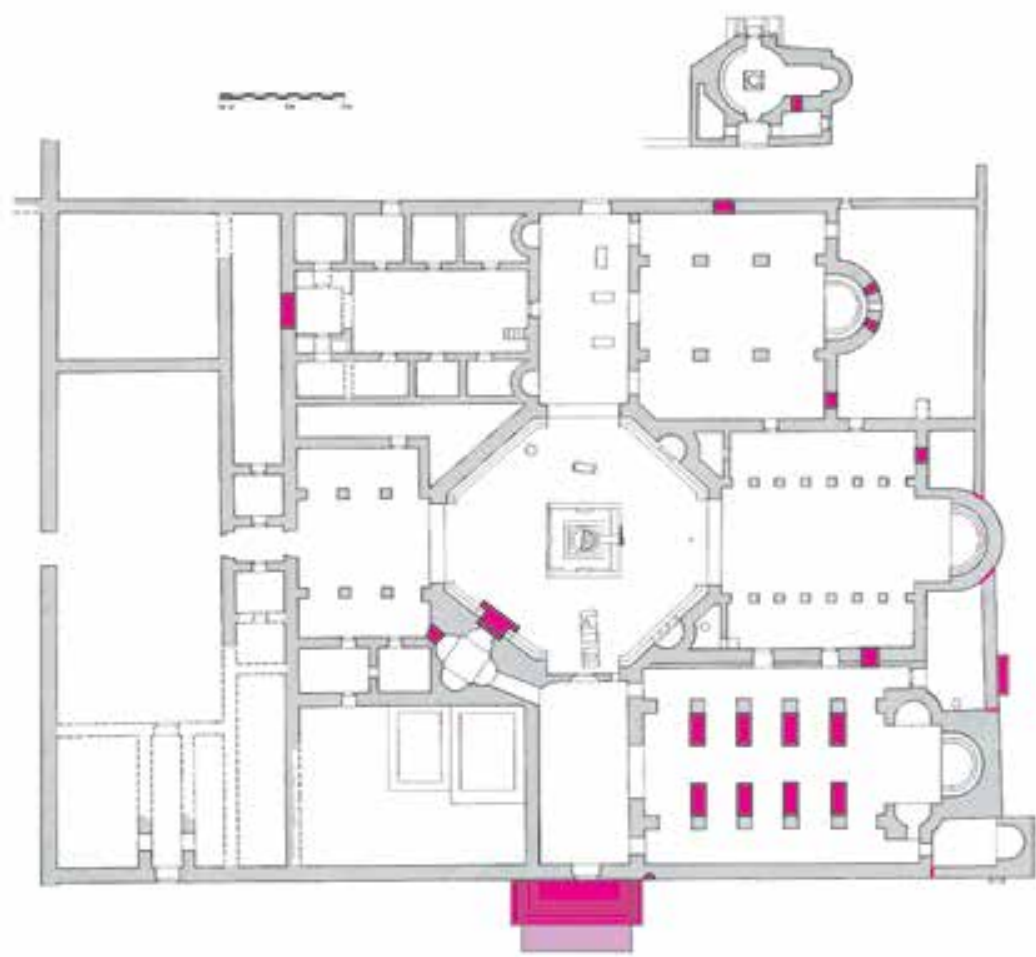

Fig. 19 - Plan de l'ensemble central du sanctuaire de Saint-Syméon-Stylite-le-Jeune avec indication des emplacements des passages bouchés et des dispositifs repérés dans les photos de Jean Mécérian, qui ont été démontés depuis (M. Gwiazda d'après DJoBADze, Archaeological Investigations in the Region West of Antioch on-the-Orontes, plan G).

- Sur le plan de Djobadze, la chapelle située dans la partie nord-est du site est privée d'abside. Or, celle-ci figure sur le plan du père Jean Mécérian, néanmoins sur la photo aérienne ses dimensions sont considérablement plus grandes. Elle est en fait aussi profonde que les pastophoria des deux côtés.

- Dans la partie ouest du sanctuaire, entre le mur intérieur et extérieur, se trouvent probablement, au moins trois pièces dont les contours sont visibles sur la photo aérienne (marqués par le trait interrompu noir et blanc). Elles figuraient sur le plan d'ensemble de Wachtang Djobadze, mais pas au bon endroit.

En outre, en se basant sur la même photo aérienne, on peut avancer quelques observations relatives aux travaux de Jean Mécérian. Une grande tache claire dans la partie sud du site indique qu'à cet emplacement se situait le principal entassement de déblais. Des entassements mineurs se laissent voir le long du mur est, au nord de 
la chapelle nord-est et dans la corne nord-ouest du mur d'enceinte intérieur. Dans l'enclos du mur intérieur, dans la partie nord du sanctuaire, on voit aussi des maisonnettes, qui servaient de base à la campagne archéologique.

$$
* * *
$$

La brève présentation de l'évolution du sanctuaire, dans la première partie du présent article, basée sur les résultats d'études architectoniques et épigraphiques publiées jusqu'à présent et sur les récits des vies de saint Syméon et de sainte Marthe, nous livre une image d'un sanctuaire en expansion qui sut conserver ses structures du $\mathrm{VI}^{\mathrm{e}}$ jusqu'au XIII ${ }^{\mathrm{e}}$ siècle. Cependant, la deuxième partie de l'article montre que les transformations survenues sur le site étaient plus complexes. L'histoire du site ne se limite pas exclusivement à la construction d'édifices successifs témoignant de son développement.

$\mathrm{Au}$ vu de la documentation d'archives que nous venons de présenter, il faut constater que dans l'enclos du sanctuaire, nous pouvons observer des changements, qui perturbèrent de manière significative le fonctionnement de ses différentes parties. Preuve en est, les nombreux bouchages réalisés avec soin et autres constructions qui, sans aucun doute, transformèrent le caractère des lieux où elles furent élevées (fig. 19). Le bouchage de l'entrée nord de la porte d'Antioche indique que la chapelle attenante domina cette partie du sanctuaire du point de vue fonctionnel, en lui ôtant sa fonction initiale, celle d'un lieu de passage. On peut supposer que cette modification pouvait être liée aux travaux de reconstruction de la porte, dans la partie ouest du mur extérieur d'enceinte. La construction des bouchages, dans différents passages de l'enclos du sanctuaire, témoigne du réaménagement des axes de communication. Nous pouvons en donner un très bon exemple en citant le bouchage situé dans le passage qui traversait le tétraconque. La création de ce couloir pouvait être liée à la transition entre la phase des débuts du culte du Stylite, qui s'exerça de son vivant autour de la colonne dans l'octogone, et le culte post-mortem organisé autour des reliques de Syméon et de sa mère déposées probablement dans l'église sud. Suite à l'ajout des murs transversaux dans la nef centrale de cette église, le culte des saints devenait considérablement plus difficile. Il en résulta un abandon ou une limitation de l'utilisation de l'édifice à ces fins. Probablement on bloqua aussi le couloir menant par le tétraconque, qui facilitait l'accès à l'église sud en contournant un passage entre l'octogone et le couloir sud, très étroit et peu confortable compte tenu d'une cage d'escalier monolithique.

Il faut tout de même souligner que la formulation de conclusions définitives, relatives aux changements constatés dans le fonctionnement des différentes parties du sanctuaire, est rendue difficile du fait qu' une chronologie précise des transformations en question est impossible à établir. 


\section{BIBLIOGRAPHIE}

CAHEN C. (1940), La Syrie du Nord à l'époque des croisades et la principauté franque d'Antioche, (Institut Français de Damas, Bibliothèque orientale, 1) Librairie orientaliste Paul Geuthner, Paris.

Callot O. (1989), «À propos de quelques colonnes de stylites syriens», in Etienne R., Le Dinahet M.-T. et Yon M. (éds.), Architecture et poésie dans le monde grec: Hommage à Georges Roux, (Collection de la Maison de l'Orient Méditerranéen, Sér. Arch., 11) Maison de l'Orient Méditerranéen, Paris, p. 107-122.

Djobadze W. (1964a), «Report on Archaeological Activities in the Vicinity of Antakya », Türk Arkeoloji Dergisi 13/1, p. 53-55.

Id. (1964b), «Second Preliminary Report on Excavations in the Vicinity of Antioch-on-theOrontes », Türk Arkeoloji Dergisi 13/2, p. 32-35.

Id. (1965), «Vorläufiger Bericht über Grabungen und Untersuchungen in der Gegend von Antiochia am Orontes », Istanbuler Mitteilungen 15, p. 218-242.

Id. (1976), Materials for the Study of Georgian Monasteries in the Western Environs of Antioch on the Orontes, (Corpus Scriptorium Christianorum Orientalium. Subsidia 48) Secrétariat du Corpus SCO, Louvain.

Id. (1986), Archaeological Investigations in the Region West of Antioch on-the-Orontes, (Forschungen zur Kunstgeschichte und Christlichen Archäologie, 13) Franz Steiner Verlag Wiesbaden, Stuttgart.

Dufay B. (1984), Immersions. Lieux et pratiques de l'initiation chrétienne dans le patriarcat d'Antioche (étude archéologique et liturgique des baptistères paléochrétiens), Paris (thèse de doctorat non publiée).

Frolow A. (1956), «IC XC NIKA », Byzantinoslavica 17/1, p. 98-113.

Gough M. (1962), « The Church of the Evangelists at Alahan: A Preliminary Report », Anatolian Studies 12, p. 173-184.

Id. (1963), «Excavations at Alahan Monastery: Second Preliminary Report », Anatolian Studies 13, p. 105-115.

Id. (1968), « Alahan Monastery: Fifth Preliminary Report », Anatolian Studies 18, p. 159-167.

Hendy M.F. (1999), Catalogue of the Byzantine Coins in the Dumbarton Oaks Collection and in the Whittemore Collection, vol. IV: Alexius I to Michael VIII: 1081-1261, Dumbarton Oaks Research Library and Collection, Washington (D.C.).

LAFONTAine-Dosogne J. (1967), Itinéraires archéologiques dans la région d'Antioche. Recherches sur le monastère et sur l'iconographie de S. Syméon Stylite le Jeune, (Bibliothèque de Byzantion, 4) Édition de Byzantion, Bruxelles.

MÉCÉRIAN J. (1934), «Une mission archéologique dans l'Antiochène. Rapport sur la deuxième campagne de fouilles 1933 », Comptes rendus des Séances de l'Académie des Inscriptions et Belles-Lettres 78/2, p. 144-149.

Id. (1948), « Monastère de Saint-Siméon-Stylite-le-Jeune, exposé des fouilles », Comptes rendus des Séances de l'Académie des Inscriptions et Belles-Lettres 92/3, p. 323-328.

Id. (1951), «Le monastère de Saint Syméon le Stylite du Mont Admirable », in Actes du $V I^{\mathrm{e}}$ Congrès international des études byzantines, Paris 27 juillet -2 août 1948, 2 vol. en 1, École des Hautes Études, Paris, vol. II, p. 299-302.

Id. (1962), «Les inscriptions du Mont Admirable», Mélanges de l'Université Saint Joseph 38, p. 297-330.

Id. (1964), «Expédition archéologique dans l'Antiochène occidentale », MUSJ 40, p. 1-144. 
Metcalf D.M. (1995), Coinage of the Crusades and the Latin East in the Ashmolean Museum, Oxford, 2nd ed., (Special Publications - Royal Numismatic Society, 28) Royal Numismatic Society - Society for the Study of the Crusades and the Latin East, Londres.

Millet G. (1933), «La mission archéologique du P. Mécérian dans l'Antiochène », Comptes rendus des Séances de l'Académie des Inscriptions et Belles-Lettres 77/3, p. 343-348.

Id. (1935), « Un rapport du R.P. Mécérian sur les fouilles au monastère de Saint-Syméon-le Jeune au Mont Admirable (Syrie) », Comptes rendus des Séances de l'Académie des Inscriptions et Belles-Lettres 79/2, p. 195-197.

Id. (1936), «Rapport du P. Mécérian sur les fouilles au monastère de Saint-Syméon le Jeune au Mont Admirable (Syrie) », Comptes rendus des Séances de l'Académie des Inscriptions et Belles-Lettres 80/3, p. 205-206.

Morray D.W. (1994), « The Defences of the Monastery of St Simeon the Younger on Samandağ », Orientalia Christiana Periodica 60/2, p. 619-623.

Morrisson C. (1970), Catalogue des monnaies byzantines de la Bibliothèque Nationale, 2 vol., vol. II: De Philippicus a Alexis III (711-1204), Bibliothèque Nationale, Paris.

Peeters P. (1928), «L'église Géorgienne du Clibanion au Mont Admirable», Analecta Bollandiana 46, p. 241-286.

PETERSON E. (1926), Eî́ Oعós. Epigraphische, formgeschichtliche und religionsgeschichtliche Untersuchungen, (Forschungen zur Religion und Literatur des Alten und Neuen Testaments, 24) Vandenhoeck \& Ruprecht, Göttingen.

Piccirillo M. (1989), Chiese e Mosaici di Madaba, (Publications of the Studium Biblicum Franciscanum, Collectio maior, 34) Franciscan Printing Press, Jerusalem.

Prentice W.K. (1908), Greek and Latin Inscriptions, Part III of the Publications of an American Archaeological Expedition to Syria 1899-1900, Century Co., New York.

Rey-CoquaIS J.-P. (1995), «Inscriptions du Mont Admirable », MUSJ 52, p. 199-226.

Trombley F.R. (2004), « Demographic and Cultural Transition in the Territorium of Antioch, $6^{\text {th }}-10^{\text {th }}$ c. », Topoi, Supplementum 5, p. 341-362.

Ulbert T. (1986), Die Basilika des Heiligen Kreuzes in Resafa-Sergiupolis, (Resafa, 2) Philipp von Zabern, Mainz am Rhein.

VAN DEN Ven P. (1961), « Le martyrium en triconque dans la vie de Sainte Marthe », Byzantion 31, p. $249-255$.

Id. (1962-1970), La vie ancienne de S. Syméon Stylite le Jeune (521-592), 2 vol., vol. I: Introduction et texte grec - vol. II : Vie grecque de sainte Marthe mère de S. Syméon, (Subsidia Hagiographica, 32) Société des Bollandistes, Bruxelles.

Verzone P. (1974), «Il santuario di S. Simeone il Giovane sul Monte Delle Meraviglie », Corsi di cultura sull'arte Ravennate e Bizantina, p. 273-286.

Zayadine F. (1994), "Ayla-'Aqaba in the Light of Recent Excavations », Annual of the Department of Antiquities of Jordan 38, p. 485-505. 